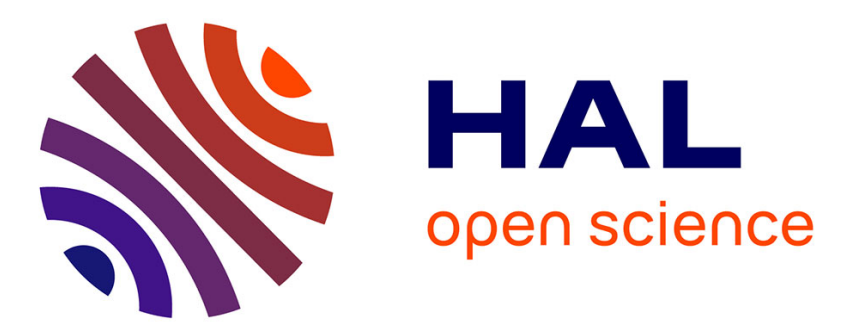

\title{
Computing least squares condition numbers on hybrid multicore/GPU systems
}

Marc Baboulin, Jack Dongarra, Rémi Lacroix

\section{To cite this version:}

Marc Baboulin, Jack Dongarra, Rémi Lacroix. Computing least squares condition numbers on hybrid multicore/GPU systems. [Research Report] RR-8479, INRIA. 2014. hal-00947204v2

\section{HAL Id: hal-00947204 \\ https://hal.inria.fr/hal-00947204v2}

Submitted on 28 Feb 2014

HAL is a multi-disciplinary open access archive for the deposit and dissemination of scientific research documents, whether they are published or not. The documents may come from teaching and research institutions in France or abroad, or from public or private research centers.
L'archive ouverte pluridisciplinaire HAL, est destinée au dépôt et à la diffusion de documents scientifiques de niveau recherche, publiés ou non, émanant des établissements d'enseignement et de recherche français ou étrangers, des laboratoires publics ou privés. 
Computing least squares condition numbers on

hybrid multicore/GPU systems

Marc Baboulin, J. Dongarra, Rémi Lacroix

RESEARCH

REPORT

$\mathrm{N}^{\circ} 8479$

Février 2014

Project-Team Postale 



\title{
inzián
}

\section{Computing least squares condition numbers on hybrid multicore/GPU systems}

\author{
Marc Baboulin*, J. Dongarra† Rémi Lacroix $\rceil^{\dagger}$ \\ Project-Team Postale \\ Research Report n ${ }^{\circ} 8479$ - Février 2014 - 8 pages
}

\begin{abstract}
This paper presents an efficient computation for least squares conditioning or estimates of it. We propose performance results using new routines on top of the multicore-GPU library MAGMA. This set of routines is based on an efficient computation of the variance-covariance matrix for which, to our knowledge, there is no implementation in current public domain libraries LAPACK and ScaLAPACK.
\end{abstract}

Key-words: linear least squares, condition number, statistical condition estimation, variancecovariance, GPU computing, MAGMA library.

* Inria and Université Paris-Sud, Orsay, France (marc.baboulin@inria.fr).

$\dagger$ University of Tennessee, Knoxville, USA (dongarra@eecs.utk.edu).

¥ Inria and Université Pierre et Marie Curie, Paris, France (remi.lacroix@inria.fr).

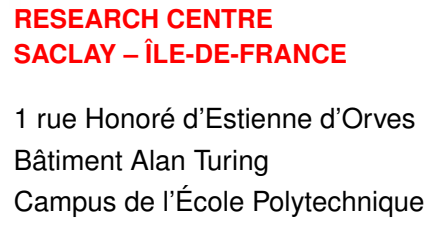




\section{Calculer les conditionnements de moindres-carrés sur systèmes multicœurs/GPU}

Résumé : Cet article présente un calcul efficace pour le conditionnement des moindres carrés ou des estimateurs de ce conditionnement. Nous proposons des résultats de performance en utilisant une nouvelle implémentation à partir de la bibliothèque logicielle pour architectures multicœurs+GPU MAGMA. Cet ensemble de programmes repose sur un calcul efficace de la matrice de variance-covariance pour laquelle, à notre connaissance, il n'y a pas d'implémentation dans les bibliothèques logicielles du domaine public LAPACK et ScaLAPACK.

Mots-clés : moindres carrés linéaires, conditionnement, estimateur statistique de conditionnement, variance-covariance, calculs sur GPU, bibliothèque MAGMA. 


\section{Introduction}

Linear least squares (LLS) is a classical linear algebra problem in scientific computing, arising for instance in many parameter estimation problems [5]. We consider the overdetermined full rank linear least squares problem $\min _{x \in \mathbb{R}^{n}}\|A x-b\|_{2}$, with $A \in \mathbb{R}^{m \times n}, m \geq n$ and $b \in \mathbb{R}^{m}$.

In addition to computing LLS solutions efficiently, an important issue is to assess the numerical quality of the computed solution. The notion of conditioning provides a theoretical framework that can be used to measure the numerical sensitivity of a problem solution to perturbations. Similarly to 2, 3] we suppose that the perturbations on data are mesured using the Frobenius norms for matrices and the Euclidean norm for vectors. Then we can derive simple formulas for the condition number of the LLS solution $x$ or its components using the $R$ factor (from the QR decomposition of $A$ ), the residual and $x$. We can also use the variance-covariance matrix.

In this paper we propose algorithms to compute LLS condition numbers in a computational time that is affordable for large scale simulations, in particular using the variance-covariance matrix. We also compute statistical condition estimates that can be obtained cheaply $\left(\mathcal{O}\left(n^{2}\right)\right.$ operations) and with a satisfying accuracy using an approach similar to [6, 8]. For these algorithms we describe an implementation for LLS conditioning using the MAGMA library 4, 10, which is a dense linear algebra library for heterogeneous multicore-GPU architectures with interface similar to LAPACK. Our implementation takes advantage of current hybrid multicore-GPU systems by splitting the computational work between the GPU and the multicore host. We present performance results and these results are compared with the computational cost for computing the LLS solution itself.

\section{Closed formulas and statistical estimates}

In this section we recall some existing formulas to compute or estimate the condition number of an LLS solution $x$ or of its components. We suppose that the LLS problem has already been solved using a QR factorization (the normal equations method is also possible but the condition number is then proportional to $\left.\operatorname{cond}(A)^{2}\right)$. Then the solution $x$, the residual $r=b-A x$, and the factor $R \in \mathbb{R}^{n \times n}$ of the $\mathrm{QR}$ factorization of $A$ are readily available.

From [3] we obtain a closed formula for the absolute condition number of the LLS solution as

$$
\kappa_{L S}=\left\|R^{-1}\right\|_{2}\left(\left\|R^{-1}\right\|_{2}^{2}\|r\|_{2}^{2}+\|x\|_{2}^{2}+1\right)^{\frac{1}{2}},
$$

where $x, r$ and $R$ are exact quantities.

We can also compute $\bar{\kappa}_{L S}$, statistical estimate of $\kappa_{L S}$ that is obtained using the condition numbers of $z_{i}^{T} x$ where $z_{1}, z_{2}, \ldots, z_{q}$ are $q$ random orthogonal vectors of $\mathbb{R}^{n}$, obtained for instance via a $\mathrm{QR}$ factorization of a random matrix $Z \in \mathbb{R}^{n \times q}$. The condition number of $z_{i}^{T} x$ can be computed using the expression given in 3] as

$$
\kappa_{i}=\left(\left\|R^{-1} R^{-T} z_{i}\right\|_{2}^{2}\|r\|_{2}^{2}+\left\|R^{-T} z_{i}\right\|_{2}^{2}\left(\|x\|_{2}^{2}+1\right)\right)^{\frac{1}{2}} .
$$

Then $\bar{\kappa}_{L S}$ is computed using the expression $\bar{\kappa}_{L S}=\frac{\omega_{q}}{\omega_{n}} \sqrt{\sum_{j=1}^{q} \kappa_{j}^{2}}$ with $\omega_{q}=\sqrt{\frac{2}{\pi\left(q-\frac{1}{2}\right)}}$. As explained in [6], choosing $q=2$ random vectors enables us to obtain a satisfying accuracy.

By considering in Equation (2) the special case where $z_{i}=e_{i}$ where $e_{i}$ is a canonical vector of $\mathbb{R}^{n}$, we can express in Equation (3) the condition number of the component $x_{i}=e_{i}^{T} x$. Then we can calculate a vector $\kappa_{C W} \in \mathbb{R}^{n}$ with components $\kappa_{i}$ being the exact condition number of $x_{i}$ and expressed by

$$
\kappa_{i}=\left(\left\|R^{-1} R^{-T} e_{i}\right\|_{2}^{2}\|r\|_{2}^{2}+\left\|R^{-T} e_{i}\right\|_{2}^{2}\left(\|x\|_{2}^{2}+1\right)\right)^{\frac{1}{2}} .
$$


We can also find in [6, 8] a statistical estimate for each $\kappa_{i}$.

\section{Variance-covariance matrix}

In many physical applications, LLS problems are expressed using a statistical model often referred to as linear statistical model where we have to solve

$$
b=A x+\epsilon, A \in \mathbb{R}^{m \times n}, b \in \mathbb{R}^{m},
$$

with $\epsilon$ being a vector of random errors having expected value 0 and variance-covariance $\sigma_{b}^{2} I$. The matrix $A$ is called the regression matrix and the unknown vector $x$ is called the vector of regression coefficients. Following the Gauss-Markov theorem [11, the least squares estimate $\hat{x}$ is the linear unbiased estimator of $x$ satisfying $\hat{x}=\arg \min _{x \in \mathbb{R}^{n}}\|A x-b\|_{2}$, with minimum variance-covariance equal to $C=\sigma_{b}^{2}\left(A^{T} A\right)^{-1}$. The diagonal elements $c_{i i}$ of $C$ give the variance of each component $\hat{x}_{i}$. The off-diagonal elements $c_{i j}, i \neq j$ give the covariance between $\hat{x}_{i}$ and $\hat{x}_{j}$. Then instead of computing condition numbers (which are notions more commonly handled by numerical linear algebra practitioners) physicists often compute the variance-covariance matrix whose entries are intimately correlated with condition numbers $\kappa_{i}$ and $\kappa_{L S}$ mentioned previously.

When the variance-covariance matrix has been computed, the condition numbers can be easily obtained. Indeed, we can use the fact that $\left\|R^{-1}\right\|_{2}^{2}=\frac{\|C\|_{2}}{\sigma_{b}^{2}},\left\|R^{-T} e_{i}\right\|_{2}^{2}=\frac{c_{i i}}{\sigma_{b}^{2}}$, and $\left\|R^{-1} R^{-T} e_{i}\right\|_{2}=$ $\frac{\left\|C_{i}\right\|_{2}}{\sigma_{b}^{2}}$ where $C_{i}$ and $c_{i i}$ are respectively the $i$ th column and the $i$ th diagonal element of the matrix $C$. Then by replacing respectively in Equations (1) and (3) we get the formulas

$$
\kappa_{L S}=\frac{\|C\|_{2}^{1 / 2}}{\sigma_{b}}\left((m-n)\|C\|_{2}+\|x\|_{2}^{2}+1\right)^{1 / 2},
$$

and

$$
\kappa_{i}=\frac{1}{\sigma_{b}}\left((m-n)\left\|C_{i}\right\|_{2}^{2}+c_{i i}\left(\|x\|_{2}^{2}+1\right)\right)^{1 / 2} .
$$

Note that, when $m>n, \frac{1}{m-n}\|r\|_{2}^{2}$ is an unbiased estimate of $\sigma_{b}^{2}$ [7, p. 4].

\section{Implementation details}

We developped a set of routines that compute the following quantities using the MAGMA library (release 1.2.1):

- Variance-covariance matrix $C$.

- $\kappa_{L S}$, condition number of $x$.

- $\kappa_{C W}$, vector of the $\kappa_{i}$, condition numbers of the solution components.

- $\bar{\kappa}_{L S}$, statistical estimate of $\kappa_{L S}$.

- $\bar{\kappa}_{C W}$, vector of the statistical estimates $\kappa_{i}$.

The variance-covariance computation requires inverting a triangular matrix and multiplying this triangular matrix by its transpose (similarly to the LAPACK routine DPOTRI [1, p. 26] that computes the inverse of a matrix from its Cholesky factorization). These operations use a block algorithm which, for the diagonal blocks, is performed recursively. The recursive part is 
performed by the CPU for sake of performance while the rest of the algorithm is executed on the GPU.

The computation of the exact condition number $\kappa_{L S}$ from the variance-covariance using Equation (4) involves the computation of the spectral norm of $C$ which is generally computed via an SVD. However, since $A$ is a full rank matrix, $C$ is symmetric positive definite and its singular values coincide with its eigenvalues. Then we use an eigenvalue decomposition of $C$ which is faster than an SVD because it takes into account the symmetry of $C$. The tridiagonalization phase is performed on the GPU while the subsequent eigenvalue computation is performed on the CPU host.

The statistical estimates require the generation and orthonormalization of random vectors followed by 2 triangular solves. The random generation and the triangular solves are performed on the GPU. The orthonormalization is performed on the CPU because it is applied to small matrices (small number of samples).

\section{$5 \quad$ Performance results}

Our experiments have been achieved on a multicore processor Intel Xeon E5645 (2 sockets $\times 6$ cores) running at $2.4 \mathrm{GHz}$ (the cache size per core is $12 \mathrm{MB}$ and the size of the main memory is $48 \mathrm{~GB}$ ). This system hosts two GPU NVIDIA Tesla C2075 running at $1.15 \mathrm{GHz}$ with 6 GB memory each. MAGMA was linked with the libraries MKL 10.3.8 and CUDA 4.1, respectively, for multicore and GPU. We consider random LLS problems obtained using the method given in 9 for generating LLS test problems with known solution $x$ and residual norm.

We plot in Fig. 1 the CPU time to compute LLS solution and condition numbers using 12 threads and 1 GPU. We observe that the computation of the variance-covariance matrix and of the components conditioning $\kappa_{i}$ are significantly faster than the cost for solving the problem with respectively a time factor larger than 3 and 2, this factor increasing with the problem size. The $\kappa_{i}$ are computed using the variance-covariance matrix via Equation (5). The time overhead between the computation of the $\kappa_{i}$ and the variance-covariance computation comes from the computation of the norms of the columns (routine cublasDnrm2) which has a non-optimal implementation. As expected, the routines SCE LLS and SCE LLS CW that compute statistical condition estimates for respectively the solution and all solution components outperform the other routines. Note that we did not mention on this graph the performance for computing $\kappa_{L S}$ using Equation (4). Indeed this involves an eigenvalue decomposition of the variance-covariance matrix (MAGMA routine magma_dsyevd_gpu), which turns out to be much slower than the LLS solution (MAGMA routine magma dgels3 gpu) in spite of a smaller number of flops $\left(\mathcal{O}\left(n^{3}\right)\right.$ vs $\left.\mathcal{O}\left(m n^{2}\right)\right)$ which shows that having an efficient implementation on the targetted architecture is essential to take advantage of the gain in flops.

We can illustrate this by comparing in Fig. 2 the time for computing an LLS solution and its conditioning using LAPACK and MAGMA. We observe that MAGMA provides faster solution and condition number but, contrary to LAPACK, the computation of the condition number is slower than the time for the solution, in spite of a smaller flops count. This shows the need for improving the Gflop/s performance of eigensolvers or SVD solvers for GPUs but it also confirms the interest of considering statistical estimates on multicore-GPU architectures to get fast computations. 


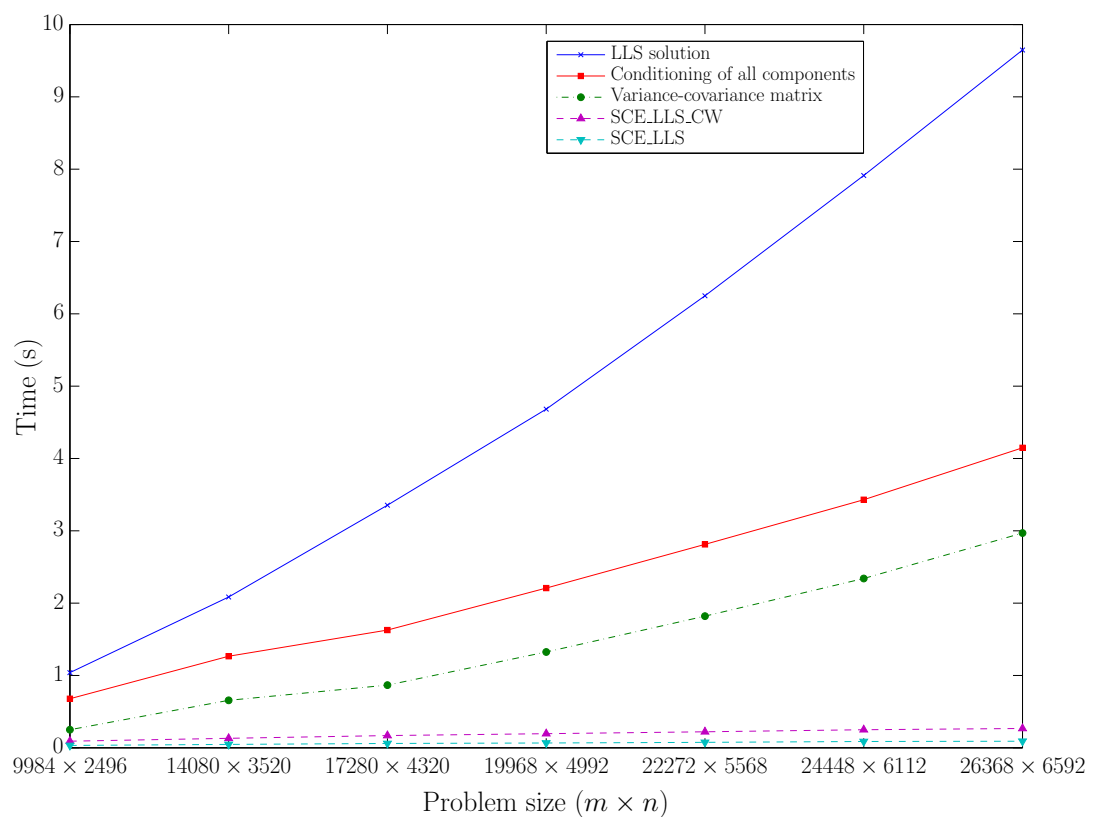

Figure 1: Performance for computing LLS condition numbers with MAGMA

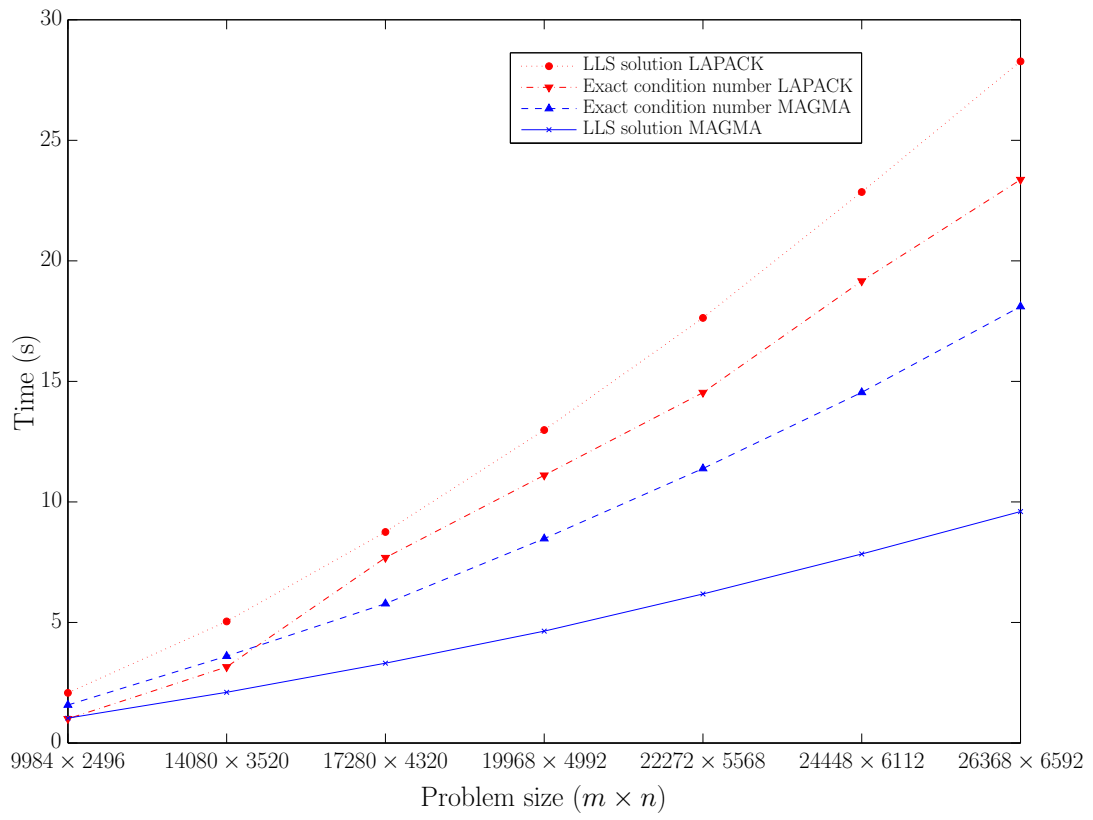

Figure 2: Time for LLS solution and condition number 


\section{Conclusion}

We proposed new implementations for computing LLS condition numbers using the software libraries LAPACK and MAGMA. The performance results that we obtained on a current multicoreGPU system confirmed the interest of using statistical condition estimates. New routines will be integrated in the next releases of LAPACK and MAGMA to compute the variance-covariance matrix after a linear regression.

\section{References}

[1] E. Anderson, Z. Bai, C. Bischof, S. Blackford, J. Demmel, J. Dongarra, J. Du Croz, A. Greenbaum, S. Hammarling, A. McKenney, and D. Sorensen. LAPACK Users' Guide. SIAM, Philadelphia, 1999. Third edition.

[2] M. Arioli, M. Baboulin, and S. Gratton. A partial condition number for linear least-squares problems. SIAM J. Matrix Analysis and Applications, 29(2):413-433, 2007.

[3] M. Baboulin, J. Dongarra, S. Gratton, and J. Langou. Computing the conditioning of the components of a linear least squares solution. Numerical Linear Algebra with Applications, 16(7):517-533, 2009.

[4] M. Baboulin, J. Dongarra, and S. Tomov. Some issues in dense linear algebra for multicore and special purpose architectures. In 9th International Workshop on State-of-the-Art in Scientific and Parallel Computing (PARA'08), volume 6126-6127 of Lecture Notes in Computer Science. Springer-Verlag, 2008.

[5] M. Baboulin, L. Giraud, S. Gratton, and J. Langou. Parallel tools for solving incremental dense least squares problems. Application to space geodesy. Journal of Algorithms and Computational Technology, 3(1):177-133, 2009.

[6] M. Baboulin, S. Gratton, R. Lacroix, and A. J. Laub. Statistical estimates for the conditioning of linear least squares problems. In Proceedings of 10th International Conference on Parallel Processing and Applied Mathematics (PPAM 2013), 2013.

[7] A. Björck. Numerical Methods for Least Squares Problems. SIAM, Philadelphia, 1996.

[8] C. S. Kenney, A. J. Laub, and M. S. Reese. Statistical condition estimation for linear least squares. SIAM J. Matrix Analysis and Applications, 19(4):906-923, 1998.

[9] C. C. Paige and M. A. Saunders. LSQR: An algorithm for sparse linear equations and sparse least squares. ACM Trans. Math. Softw., 8(1):43-71, 1982.

[10] S. Tomov, J. Dongarra, and M. Baboulin. Towards dense linear algebra for hybrid GPU accelerated manycore systems. Parallel Computing, 36(5\&6):232-240, 2010.

[11] M. Zelen. Linear estimation and related topics. In J.Todd, editor, Survey of numerical analysis, pages 558-584. McGraw-Hill, New York, 1962. 


\section{Contents}

$\begin{array}{lll}1 & \text { Introduction } & 3\end{array}$

\begin{tabular}{|lll}
2 & Closed formulas and statistical estimates & 3
\end{tabular}

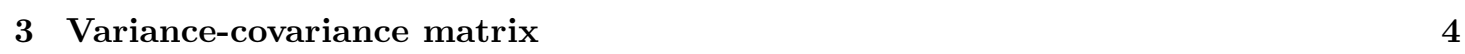

\begin{tabular}{|lll}
\hline & Implementation details & 4
\end{tabular}

$\begin{array}{lll}5 & \text { Performance results } & 5\end{array}$

\begin{tabular}{lll}
\hline 6 & Conclusion & 7
\end{tabular} 


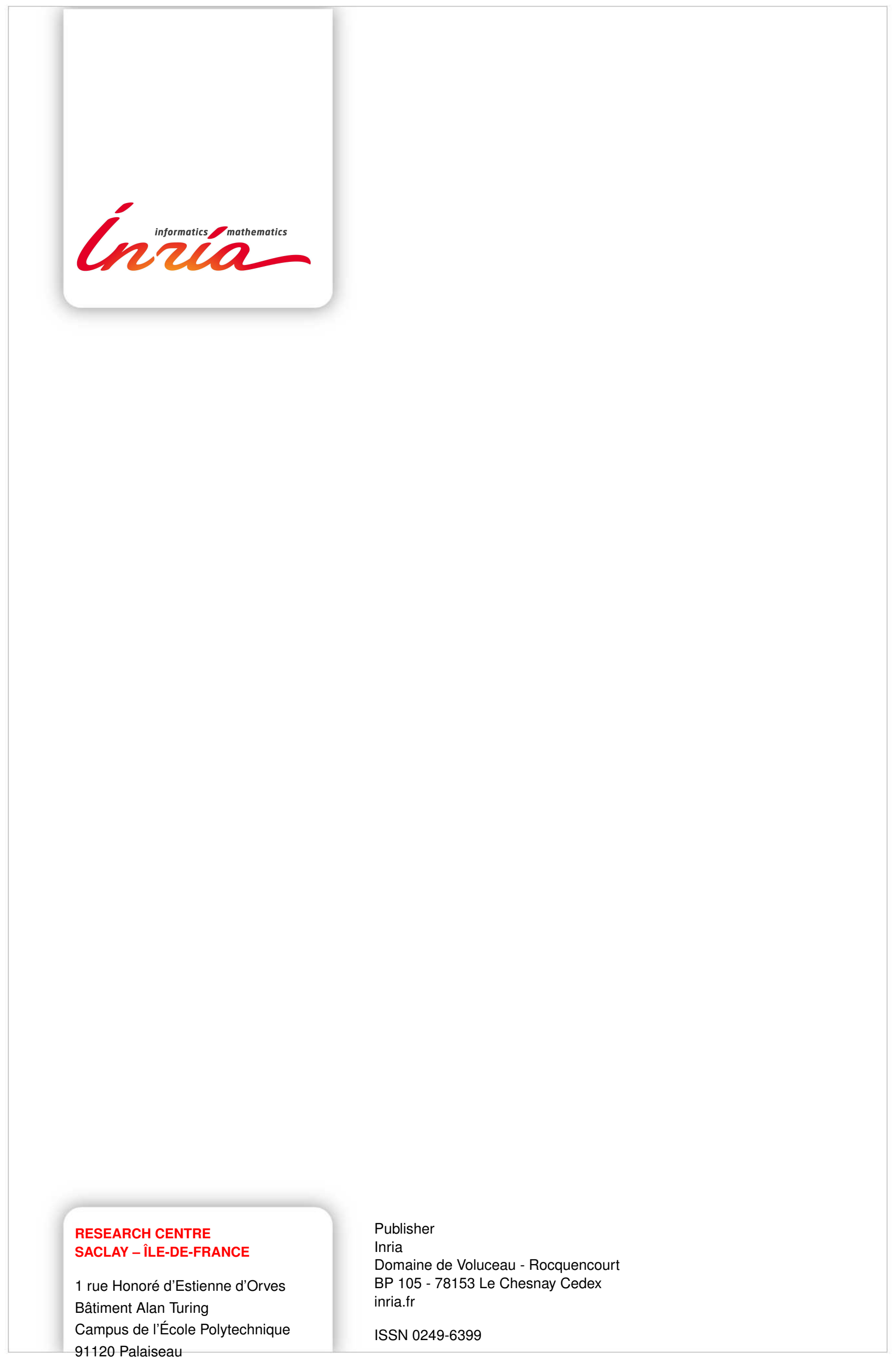

